H I G H L I G H T S

\section{Inviting malaria in}

Erythrocytes, which are incapable of endocytosis, can be infected by the malaria parasite Plasmodium falciparum. How this parasite gets into erythrocytes is a long-standing puzzle. Now, in a study published in Science, Harrison et al. link signalling by host (erythrocyte) proteins to the entry of $P$. falciparum and other malarial parasites.

In the first step of erythrocyte invasion the malaria parasite is internalised into a vacuole - the parasite vacuole - formed by the erythrocyte membrane. Multiplication of malarial parasites inside erythrocytes, and the release of parasitic waste products, produces the episodic chills and fever that characterize malaria. Previous work from the same laboratory showed that a host heterotrimeric $\mathrm{G}$ protein - $\mathrm{G} \alpha_{\mathrm{s}}$, one of three $\mathrm{G}$ proteins in erythrocytes - enriched in cholesterol-rich membrane rafts was specifically recruited to the parasite vacuolar membrane. Heterotrimeric G proteins are signal transduction proteins in eukaryotes, which are activated by binding to receptors called G-protein coupled receptors (GPCRs) and transduce extracellular information (such as hormones) to activate cellular proteins. But their function in erythrocytes is poorly understood. Here, Harrison and co-workers show that the erythrocyte GPCR that $G \alpha_{\text {s }}$ interacts with $-\beta-\mathrm{AR}-$ is also recruited to the parasite vacuole.

Specifically blocking activation of $\mathrm{G} \alpha_{\mathrm{s}}$ using synthetic peptides matching $\mathrm{G} \alpha_{\mathrm{s}}$ sequences that compete for interaction with $\beta$-AR led to a dramatic reduction in erythrocyte infection by $P$. falciparum. Stimulation of GPCRs (to activate $\mathrm{G} \alpha_{\mathrm{s}}$ ) enhanced parasite infection of erythrocytes. Strikingly, inhibiting activation of $\mathrm{G} \alpha_{\mathrm{s}}$ in a mouse model for malaria (Plasmodium berghei) reduced parasite infection. Activating $G \alpha_{s}$ promotes erythrocyte invasion by malaria, and this mechanism is conserved across malaria species.

The link between host cell signals and regulating establishment of the parasite vacuole is clear. The next big hurdles are finding out how $\mathrm{G} \alpha_{\mathrm{s}}$-coupled receptors are stimulated in the first place and which effector molecules come in to play downstream of $\mathrm{G} \alpha_{\mathrm{s}}$ in erythrocytes. The authors speculate that catecholamines produced during infection could switch on $\mathrm{G} \alpha_{\mathrm{s}}$ - coupled receptors which in turn signal to rearrange the cytoskeleton and help the parasite to invade the erythrocyte. Whether modification of $\mathrm{G} \alpha_{\mathrm{s}}$ regulates other erythrocyte membrane functions which could relate to diseases like haemolytic anaemias — remains to be seen.

\section{(D) References and links} Susan Jones ORIGINAL RESEARCH PAPER Harrison, T. et al. Erythrocyte G Protein-coupled receptor signaling in malarial infection. Science 301, 1734-1736 (2003) FURTHER READING Haldar, K. et al. Protein and lipid trafficking induced in erythrocytes infected by malaria parasites. Cell Microbiol. 4, 383-395 (2002) WEB SITE

Kasturi Haldar's laboratory:

http://bugs.mimnet.northwestern.edu/labs/Faculty haldark.html

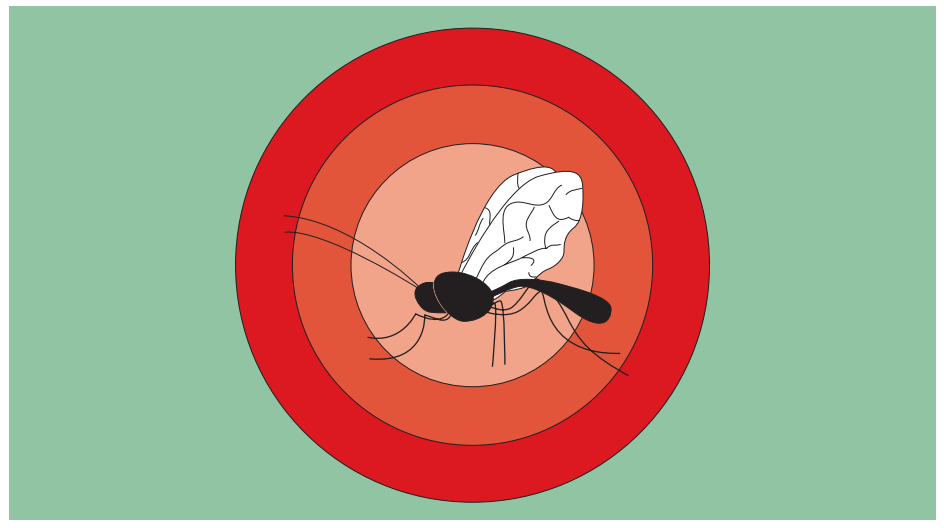

IN BRIEF

VACCINES

Construction and evaluation of a safe, live, oral Vibrio cholerae vaccine candidate, IEM108

Liang, W. et al. Infect. Immun. 71, 5498-5504 (2003)

Vaccination against cholera is a feasible prevention strategy but because toxin genes are present on a lysogenic Vibrio bacteriophage (CTX $\Phi$ ), any live attenuated vaccine strain should ideally be phage resistant. Otherwise, phage infection could make a safe vaccine strain toxigenic. A new candidate vaccine strain - IEM008 - incorporates a gene that represses the replication and integration functions of the CTX $\Phi$ phage. IEM008 protected rabbits against challenge with 4 wild-type strains of $V$. cholerae in rabbits and is an excellent candidate for field tests.

\section{FUNGAL ECOLOGY}

\section{Seasonal dynamics of previously unknown fungal} lineages in tundra soils

Schadt, C. W. et al. Science 301, 1359-1361 (2003)

Exciting findings have revealed that undersnow soils are physiologically active. The most surprising outcome of this study was that huge numbers of mainly novel fungi accounted for most of the active biomass. The community structure changed dramatically between summer and winter months, but fungal activity dominated throughout. New lineages of identified fungi will undoubtedly revolutionize our knowledge of tundral ecosystems.

\section{SYMBIOSIS}

\section{LysM domain receptor kinases regulating rhizobial Nod} factor-induced infection

Limpens, Eet al. Science Aug 282003 (doi:10.1126/science.1090074)

Rhizobial bacteria establish symbiotic nitrogen-fixing nodules on legume roots in a strictly host-specific manner. Limpens et al. identified 2 genes in a model legume - LYK3/4 - that were essential for establishing nodules. Rhizobial species export socalled Nod factors, which induce host plant nodulation. The authors speculate that LysM domains - known to recognize peptidoglycan-like structures - in LYK3/4 discriminate between different Nods.

\section{BACTERIAL SECRETION}

Structural determinants of SecB recognition by SecA in bacterial protein translocation

Zhou, J. \& Xu, Z. Nature Struct. Biol. Sept 282003 (doi:10.1038/nsb980)

Secretion across the Gram-negative bacterial inner membrane is mainly through the Sec system. New structural information for $\mathrm{SecB}-\mathrm{a}$ cytosolic chaperone that delivers proteins to the secretion machine SecA - shows that a SecA zinc-binding motif is crucial for the SecA-SecB interaction. They propose that a SecB tetramer is positioned beneath SecA to deliver proteins to the SecA machine. 